Revue de droit comparé du travail et de la sécurité sociale

2| 2018

Harcèlement moral au travail en jurisprudence

comparée

\title{
Le régime québécois de protection contre le harcèlement psychologique au travail : particularités et enjeux jurisprudentiels
}

Julie Bourgault et Anne-Marie Laflamme

\section{(2) OpenEdition}

Édition électronique

URL : https://journals.openedition.org/rdctss/1943

DOI : $10.4000 /$ rdctss. 1943

ISSN : 2262-9815

Éditeur

Centre de droit comparé du travail et de la sécurité sociale

Édition imprimée

Date de publication : 1 juin 2018

Pagination : 22-29

ISSN : $2117-4350$

Référence électronique

Julie Bourgault et Anne-Marie Laflamme, « Le régime québécois de protection contre le harcèlement psychologique au travail : particularités et enjeux jurisprudentiels », Revue de droit comparé du travail et de la sécurité sociale [En ligne], 2 | 2018, mis en ligne le 01 novembre 2021, consulté le 13 novembre 2021. URL : http://journals.openedition.org/rdctss/1943 ; DOI : https://doi.org/10.4000/rdctss.1943

\section{(c) $(1) \odot$}

Revue de droit comparé du travail et de la sécurité sociale est mise à disposition selon les termes de la Licence Creative Commons Attribution - Pas d'Utilisation Commerciale - Pas de Modification 4.0 International. 


\section{LE RÉGIME QUÉBÉCOIS DE PROTECTION CONTRE LE HARCĖLEMENT PSYCHOLOGIQUE AU TRAVAIL: PARTICULARITÉS ET ENJEUX JURISPRUDENTIELS}

Professeure agrégée, Université du Québec en Outaouais, membre du Centre de recherche interuniversitaire sur la mondialisation et le travail (CRIMT) et de l'Équipe de recherche travail et société (ERTS).

\section{ANNE-MARIE LAFLAMME ${ }^{1}$}

Professeure titulaire et doyenne, Faculté de droit, Université Laval; membre du Centre de recherche interuniversitaire sur la mondialisation et le travail (CRIMT).

ntroduit en 2004 dans la Loi sur les normes du travail (LNT ci-après), le régime particulier de protection du harcèlement psychologique au Québec, s'ajoute au cadre légal préexistant ${ }^{2}$. D'ordre public, les dispositions contenues dans la LNT définissent le harcèlement psychologique en milieu de travail et obligent les employeurs sous juridiction québécoise à le prévenir et à le faire cesser, que le présumé harceleur soit un représentant de l'employeur ou un collègue de travail. Ce recours est accessible aux salariés couverts par la LNT, peu importe l'endroit où ils exécutent leurs tâches et peu importe la source de harcèlement ${ }^{3}$. Toutefois, dès lors que l'employeur a rempli son obligation de prévenir et de faire cesser le harcèlement, ce recours devient sans fondement, et ce, même s'il est démontré qu'il s'agit de harcèlement.

Malgré la présence d'une protection législative particulière et la reconnaissance du caractère fondamental de ce droit ${ }^{4}$, le harcèlement psychologique au travail est toujours bien présent au Québec et suscite une jurisprudence florissante. D'ailleurs, la Commission des normes, de l'équité, de

1 L'auteure remercie Clara Morissette, étudiante au baccalauréat en droit à l'Université Laval, pour son aide à la recherche jurisprudentielle.

2 Voir J. Bourgault, Le harcèlement psychologique au travail : Les nouvelles dispositions de la Loi sur les normes et leur intégration dans le régime préexistant, Montréal, Wilson \& Lafleur, 2006, pp. 7-10 et 134.

3 M.-F. Chabot, «Les normes légales contre le harcèlement psychologique : loin d'être minimales ", Conférence des juristes de l'État 2011, Actes de la XIXe Conférence tenue à Québec les 13 et 14 avril 2011, Cowansville, Yvon Blais, 2011, 483-548, p. 487-493.

4 Syndicat des employées et employés de métiers d'Hydro-Québec, section locale 1500 (SCFP-FTQ) c. Fontaine, 2006 QCCA 1642, par. 81. 
la santé et de la sécurité du travail (CNESST)5 a traité 3617 plaintes en 2016, dont 151 ont donné lieu à une intervention judiciaire ${ }^{6}$.

À ce titre, l'analyse de la jurisprudence récente du Tribunal administratif du travail (TAT ci-après), de son prédécesseur la Commission des relations de travail (CRT ci-après) ainsi que des Tribunaux d'arbitrage (TA ci-après) permet d'identifier certains enjeux jurisprudentiels. D'une part, l'identification du harcèlement psychologique au travail a donné lieu à une abondante jurisprudence qui cerne les contours de la définition législative. D'autre part, le choix du législateur d'inscrire le recours dans le régime des conditions minimales de travail n'est pas sans complexifier le processus d'indemnisation des victimes, particulièrement pour celles qui ont subi une atteinte à leur santé dont la réparation est régie exclusivement par le régime d'indemnisation des lésions professionnelles ${ }^{7}$. Le format de cette contribution ne nous permet pas de couvrir tous ces aspects. Aussi, nous nous limiterons à l'examen de la jurisprudence portant sur la notion de harcèlement psychologique au sens de la LNT (I), afin d'en montrer les contours et les limites (II).

\section{I - L'IDENTITFICATION DU HARCÈLEMENT PSYCHOLOGIQUE}

L'article 81.18 de la LNT définit ainsi la notion:

[...] une conduite vexatoire se manifestant soit par des comportements, des paroles, des actes ou des gestes répétés, qui sont hostiles ou non désirés, laquelle porte atteinte à la dignité ou à l'intégrité psychologique ou physique du salarié et qui entraîne, pour celui-ci, un milieu de travail néfaste. Une seule conduite grave peut aussi constituer du harcèlement psychologique si elle porte une atteinte telel qu'elle produit un effet nocif continu pour le salarié. À la lumière de cette définition, il y a harcèlement psychologique au travail en présence d'une conduite vexatoire produisant des effets sur le salarié et le milieu de travail. La jurisprudence définit chacune de ces composantes (A) et utilise une approche globale dans l'identification de la conduite vexatoire (B).

\section{A - LES COMPOSANTES DE LA DÉFINITION : LA CONDUITE VEXATOIRE ET SES EFFETS}

La preuve du harcèlement psychologique au travail exige d'abord d'établir le caractère vexatoire de la conduite, pour ensuite démontrer les effets de cette conduite. Sans conduite vexatoire, il n'y a pas d'effets, donc pas de harcèlement ${ }^{8}$.

5 La CNESST remplace l'ancienne Commission des normes du travail (CNT).

6 CNESST, Rapport annuel de gestion 2016, Annexe 2, p. 172 http://www.cnesst.gouv.qc.ca/Publications/400/Documents/DC400-2032-10web.pdf

7 J. Nadeau, Le harcèlement psychologique au travail : l'accès difficile à l'indemnisation, Cowansville, Yvon Blais, 2014 ; A.-M. Laflamme, J. Nadeau, « Le harcèlement psychologique au travail : une atteinte à la santé ou à la dignité de la personne? ", Conférence des juristes de l'État 2011, Actes de la XIXe Conférence tenue à Québec les 13 et 14 avril 2011, Cowansville, Yvon Blais, 2011, pp. 437-482.

8 Breton c. Compagnie d'échantillons National Itée, 2006 QCCTR 0601, par. 150.6 ; Syndicat de l'enseignement de la Rivière-du-Nord c. Commission scolaire de la Rivière-du-Nord, 2016 QCTA 653, par. 116-117; Association des professeurs de Lignery c. Commission scolaire des Grandes Seigneuries, 2017 QCTA 409, par. 601 ; Laberge et Le Havre des femmes, 2017 OCTAT 271, par. 27 ; Guimond c. Union canadienne des Moniales de l'Ordre de Saint-Ursule, 2017 QCTAT 5543, par. 62. 
La conduite vexatoire s'entend $d^{\prime}$ ' une attitude ou de comportements qui blessent ou humilient la personne dans son amour propre " ${ }^{9}$. En ce sens, le " caractère vexatoire fait référence au résultat de la conduite », «la personne sera diminuée, dénigrée tant sur le plan personnel que professionnel $»^{10}$. L'intention de nuire du harceleur n'est toutefois pas une considération pertinente à l'analyse ${ }^{11}$.

La conduite vexatoire se manifeste par des comportements, paroles, actes ou gestes hostiles ou non désirés. La jurisprudence a établi que le comportement de "quelqu'un qui manifeste des intentions agressives, qui se conduit en ennemi, de façon belliqueuse, antagoniste, adverse, défavorable ou menaçante », peut être qualifié d'hostile. Le caractère non désiré se réfère quant à lui à « un comportement qui n'a pas été recherché, voulu ou souhaité, ni explicitement ni implicitement " ${ }^{12}$. Que le refus soit implicite ou explicite, il importe que "les faits reprochés [soient] objectivement [...] perçus comme non désirables ${ }^{13}$. Ainsi, lorsque les comportements reprochés surviennent en réaction à un comportement provocateur ou dérangeant de la part du plaignant, " [I]a réaction dans ce cas, à moins d'être disproportionnée, et toujours selon les circonstances, ne peut être considérée hostile ou non désirée $»^{14}$.

Par ailleurs, puisqu'« il s'agit généralement de paroles, de gestes ou d'actes qui sont anodins lorsque pris isolément, mais dont l'effet cumulatif est sérieux à cause de leur caractère répétitif et continu ainsi que des conséquences qu'ils entraînent $»^{15}$, ils doivent se répéter pour constituer une conduite vexatoire, à moins d'être en présence d'une conduite grave. Une telle conduite grave est plutôt exceptionnelle. II s'agira, à titre d'exemples, de

9 Centre hospitalier régional de Trois-Rivières (Pavillon St-Joseph) c. Syndicat professionnel des infirmières et infirmiers Mauricie/Cœur-du-Québec, [2006] R.J.D.T. 397 (T.A.), par. 166. Voir aussi Laberge, ibid, par. 140 ; Association des professeur(e)s de Bishop's University et Université Bishop's, 2016 QCTA 743, par. 592.

10 G. Poirier, R L. Rivert, Les nouvelles normes de protection en cas de harcèlement psychologique au travail : une approche moderne, Éditions Yvon Blais, 2004, 155 pages, p. 57 et 58, cités dans Bangia c. Nadler Danino S.E.N.C., 2006 QCCRT 0419 et Danies c. Villa-Maria, 2015 QCCRT 0705.

11 Centre hospitalier régional de Trois-Rivières, supra note 8, Alliance du personnel professionnel et technique de la santé et des services sociaux (APTS) c. CIUSSS du Centre-Ouest-de-l'îlede-Montréal, 2017 QCTA 348, par. 70 ; Lachapelle-Welman c. 3233430 Canada inc. (Portes et fenêtres ADG), 2016 QCTAT 3557, par. 110. Voir par contre dans Laberge, supra note 7, § 80 dans laquelle l'intention semble être prise en compte: "Vu ce qui précède, le Tribunal conclut que les différentes situations invoquées par la demanderesse au soutien de sa plainte pour harcèlement psychologique ne démontrent pas de comportements visant à lui nuire, la blesser ou la dénigrer ». (souligné par nous)

12 Centre hospitalier régional de Trois-Rivières, supra note 8, par. 172-174. Voir au même effet: Laberge, supra note 7, par. 143 ; Bangia c. Nadler Danino, supra note 9, par. 85 ; Association des professeur(e)s de Bishop's University, supra note 8, § 594-595.

13 Syndicat des chargées et chargés de cours de l'Université de Montréal c. Université de Montréal, 2017 OCTA 225, par. 131, citant Habachi c. Commission des droits de la personne, D.T.E. 99T-945 (C.A.).

14 Syndicat de l'enseignement de la Rivière-du-Nord, supra note 7, par. 166.

15 Centre hospitalier régional de Trois-Rivières, supra note 8, par. 169. Voir aussi Bangia c. Nadler Danino, supra note 9, par. 81; Danies, supra note 9. 
" la lecture publique de reproches injustifiés à l'endroit de la plaignante devant une salle pleine de citoyens et de journalistes » ou encore de la menace "I'm gonna to kill you » adressée à la salariée ${ }^{16}$.

Pour constituer du harcèlement psychologique, une conduite vexatoire doit porter atteinte à la dignité ou à l'intégrité du salarié en plus d'entraîner un milieu de travail néfaste. L'atteinte à l'intégrité « doit laisser des marques, des séquelles qui, sans nécessairement être physiques ou permanentes, dépassent un certain seuil »; elle doit " affecter de façon plus que fugace l'équilibre physique, psychologique ou émotif de la victime ${ }^{17}$. La dignité se réfère " au respect, à l'estime de soi et à l'amour-propre d'une personne " ${ }^{18}$; elle " est bafouée lorsque des personnes ou des groupes sont marginalisés, mis de côté et dévalorisés ${ }^{19}$. Ont par exemple été considérés comme une telle atteinte des menaces de mort $^{20}$ et des propos humiliants ou méprisants ${ }^{21}$. À ce titre, une conduite jugée vexatoire portera généralement atteinte à la dignité puisqu'elle réfère également au résultat de la conduite sur l'amour-propre de la personne.

Enfin, la jurisprudence a qualifié le milieu de travail néfaste « un milieu qui est nuisible, malsain, dommageable et qui ne permet pas la réalisation des objectifs liés au contrat de travail de façon saine ${ }^{22}$. À titre illustratif, dans l'affaire Lachapelle-Welman, le TAT a jugé qu'en interrogeant le plaignant, un homme timide, à plusieurs reprises sur des sujets osés, sexuels, personnels, douteux et de mauvais goût, il n'a pas été " traité avec pudeur, discrétion, retenue, égards, estime, considération, respect, déférence et de façon respectueuse " par ses collègues de travail et qu'il a été "marginalisé et dévalorisé en raison de sa différence ${ }^{23}$, ce qui constitue un milieu de travail néfaste.

16 Dubé c. Lochaber-Partie-ouest (Municipalité du canton de), 2015 QCCRT 0455, § 196 et Franche et Restaurant \& Délicatesse Gerry's inc., 2015 QCCRT 0608.

17 Québec (Curateur public) c. Syndicat national des employés de l'hôpital St-Ferdinand, [1996] 3 R.C.S. 211 ; Centre hospitalier régional de Trois-Rivières, supra note 8, par. 177 ; Bangia c. Nadler Danino, supra note 9, par. 89 ; Laberge, supra note 7, §145.

18 Laberge, ibid, par. 145; Breton, supra, note 7, § 155 ; Law c. Ministre de l'emploi et l'immigration, [1999] 1 R C S 497, 530.

19 Law c. Ministre de l'emploi et l'immigration, ibid, 530 ; Bangia c. Nadler Danino, supra note 9, par. 88.

20 Roc c. Poulbec inc., 2007 QCCRT 0441 ; Franche, supra note 15.

21 Orentes Silva c. 9009-1729 Québec inc., 2016 QCTAT 2155; Dubé, supra note 15, §216-219.

22 Laberge, supra note 7, par. 147 ; Lachapelle-Welman, supra note 10, par. 114, Bangia c. Nadler Danino, supra note 9, §91.

23 Lachapelle-Welman, ibid., §112. 


\section{B - L'APPROCHE GLOBALE DE LA CONDUITE VEXATOIRE}

La conduite vexatoire résultant généralement de comportements répétés, les tribunaux utilisent une approche globale pour déterminer le caractère vexatoire : " II ne faut pas se limiter à les examiner au cas par cas. Cet examen demeure pertinent et nécessaire, mais l'analyse globale permet d'évaluer le degré réel de gravité de l'ensemble des conduites ${ }^{24}$.

Cette approche globale a permis à la Cour d'appel de considérer comme du harcèlement psychologique la conduite d'un salarié qui, successivement, porte atteinte à plusieurs victimes, et ce, même si chacune d'entre elles n'a pas nécessairement fait l'objet, de façon individuelle, de gestes vexatoires répétés ${ }^{25}$. À l'inverse, cette approche globale permettra, dans certains cas, de soustraire des comportements à la notion de harcèlement psychologique. Par exemple, le TAT a décidé que le fait d'avoir pris la demanderesse par le bras pour la conduire là où elle refusait d'aller et de l'avoir insultée devant sa supérieure immédiate constituaient des comportements hostiles ou non désirés. Toutefois, il a jugé qu'il ne s'agissait pas pour autant d'une conduite vexatoire puisque «[c]es deux comportements, certes déplacés, s'inscrivent plutôt dans le cadre d'une relation difficile entre des collègues de travail aux personnalités peu compatibles $»^{26}$.

Dans quelques décisions, bien qu'il se réclame de l'approche globale, le décideur évalue le caractère vexatoire de chacune des conduites plutôt que d'en évaluer le caractère hostile ou non désiré. En fait, une analyse globale implique d'abord d'examiner au cas par cas les comportements pour vérifier s'ils présentent un caractère hostile ou non désiré. Ensuite, il faut évaluer si, dans une perspective globale, ces comportements présentent le caractère répétitif essentiel à la qualification d'une conduite vexatoire ${ }^{27}$. En effet, un acte objectivement non désirable n'est pas pour autant vexatoire ${ }^{28}$. Par exemple, dans l'affaire Danies ${ }^{29}$, la CRT estime que la colère de la directrice à l'égard de la plaignante ne constituait pas en soi une conduite vexatoire et ne devait donc pas être retenue dans l'analyse globale, alors qu'il est possible de qualifier cette conduite d' " objectivement non désirable ». C'est par la répétition d'actes hostiles et non désirés, qui pris isolément peuvent paraître anodins, que la conduite devient vexatoire. D'ailleurs, dans l'affaire LachapelleWelman, chaque évènement, pris isolément, ne pouvait être qualifié de conduite vexatoire, bien qu'il s'agisse de comportements objectivement non désirables. La répétition de ces évènements a toutefois amené le TAT à conclure à une conduite vexatoire ${ }^{30}$.

24 Breton, supra note 7, §152.1 et 150.5 ; Bangia c. Nadler Danino, supra note 9, §83 ; Syndicat de l'enseignement de la Rivière-du-Nord, supra note 7, §121.

25 Centre de réadaptation en déficience intellectuelle du Saguenay-Lac-Saint-Jean (CRDI) c. Fortier, 2014 QCCA 1581.

26 Brochu c. 9139-8891 Québec inc. (Expédia Centre de croisières de Québec), 2017 QCTAT 3083.

27 Voir l'application de la démarche dans Breton, supra note 7, § 150.7 et 162-196.

28 Par exemple dans l'affaire Syndicat de l'enseignement de la Rivière-du-Nord, supra note 7, au §128, l'arbitre énonce que « [c]ertaines allégations doivent d'emblée être rejetées comme étant des gestes vexatoires posés par la direction envers le plaignant », en précisant toutefois au §145: "Quelle que soit la façon d'analyser ces situations, une par une ou globalement, ou dans la foulée d'autres événements, il n'y a rien qui permet d'y voir des gestes vexatoires de la part de la direction. ». Voir aussi, Giroux c. Café de la Débrouille, 2015 QCCRT 0590, par. 26 ; Danies, supra note 9, §614-616.

29 Danies, ibid, §614.

30 Lachapelle-Welman, supra note 10. 


\section{II - L'EXCLUSION DE LA QUALIFICATION DE HARCÈLEMENT PSYCHOLOGIQUE}

Afin de déterminer si la conduite vexatoire constitue du harcèlement psychologique, les tribunaux ont élaboré certains principes. D'une part, la conduite doit être appréciée dans la perspective d'une personne raisonnable (A). D'autre part, la jurisprudence a identifié des situations qui ne sont généralement pas du harcèlement, mais qui « pourront être perçues comme tel par une personne qui affiche une méfiance excessive, une tendance à la victimisation, une dépression, ou autre trait de personnalité ou maladie qui a pour effet de déformer la perception des choses ${ }^{31}$. C'est le cas des rapports sociaux difficiles (B) et de l'exercice normal des droits de direction de l'employeur (C).

\section{A - LA PERSPECTIVE DE LA VICTIME RAISONNABLE}

En raison du caractère subjectif de l'appréciation du harcèlement psychologique au travail, la perception du plaignant comme seul point d'analyse n'est pas appropriée ${ }^{32}$. En effet, la perception des évènements peut être teintée par la personnalité du plaignant, particulièrement lorsqu'il s'agit " d'une victime ou d'une personne ayant des problèmes de victimisation ou souffrant de paranoïa $»^{33}$. Ainsi, «toute situation impliquant la moindre contrariété dans un milieu de travail » pourrait être une manifestation de harcèlement psychologique aux yeux d'une personne qui a une vision déformée de la réalité ${ }^{34}$.

Afin d'éviter que tout propos déplacé ne soit interprété comme du harcèlement psychologique au travail ${ }^{35}$, l'analyse doit plutôt se faire à partir du modèle " subjectifobjectif " de la victime raisonnable, en déterminant si une " personne raisonnable normalement diligente et prudente, qui, placée dans les mêmes circonstances que la victime, estimerait que le présumé harceleur manifeste une conduite vexatoire $»^{36}$. Par exemple, dans l'affaire Roblin, le TAT est d'avis que la perspective du plaignant n'est pas celle d'une victime raisonnable en raison de son « incapacité [...] à voir les choses de

31 Syndicat de l'enseignement Rivière-du-Nord, supra note 7, §120 ; Syndicat des techniciens, techniciennes et professionnels, professionnelles du Centre de santé et de services sociaux de l'Énergie c. Centre de santé et de services sociaux de l'Énergie (CIUSSS), 2017 QCTA 409, $\S 982-984$.

32 Bangia c. Nadler Danino, supra note 9, §95, citant Habachi, supra note 12.

33 Ibid; Bangia c. Avoman, 2016 QCTAT 7255. M.-F. Hirigoyen, Le harcèlement moral dans la vie professionnelle, Éd. La découverte et Syros, Paris, 2001, 444 pages, p. 83 et 84, explique le phénomène de la victimisation comme suit : « certaines personnes peuvent se complaire dans une position de victime. Dans ce cas, elles ne cherchent pas à trouver une issue à leur situation difficile, car cela leur confère une identité et une occasion de se plaindre. Cette position victimaire a donné un sens à leur mal de vivre et, pour maintenir ce mode d'existence, il leur faudra poursuivre sans fin leur agresseur afin d'obtenir une réparation qui s'avérera toujours insuffisante ».

34 Bangia c. Avoman, ibid, par. 85 ; Danies, supra note 9 ; Bangia c. Nadler Danino, supra note 9, $\$ 96$.

35 Duclos et Industrielle Alliance, assurances et services financiers inc., 2015 QCCRT 0262, Requête en révision judiciaire rejetée, 2015 QCCS 6268, § 85.

36 Breton, supra note 7, paragr. 151 ; Dubé, supra note 15 , $\$ 148$ et 149 ; Guimond, supra note 7, §62 ; Lachapelle-Welman, supra note 10 ; Laberge, supra note 7, §149, Syndicat de l'enseignement de la Rivière-du-Nord, supra note 7, §118-119. 
façon correcte et raisonnable $»^{37}$. De même, dans l'affaire Laberge, le TAT considère la forte personnalité de la plaignante dans l'appréciation des évènements ${ }^{38}$.

\section{B - LES RAPPORTS SOCIAUX DIFFICILES}

C'est donc la perspective de la victime raisonnable qui permet aux tribunaux de distinguer le harcèlement psychologique des rapports sociaux difficiles. À ce titre, la jurisprudence considère que «les relations dans un milieu de travail ne sont pas forcément exemptes de débordement, d'accrochage ou de manifestation de colère ${ }^{39}$, et que "le manque d'amabilité, la froideur, l'indifférence pour autrui, la limitation des communications à ce qui est nécessaire pour l'accomplissement du travail, l'impolitesse même, sont des actes désagréables, parfois fâchants, mais qui ne débouchent pas nécessairement sur une conduite vexatoire ${ }^{40}$. Par exemple, des échanges entre deux personnes qui ne s'entendent pas, mais qui réussissent tout de même à faire entendre leurs arguments ${ }^{41}$ ou des commentaires irrespectueux s'inscrivant dans un contexte de guerre ouverte, n'ont pas été considérés comme du harcèlement. Dans ce dernier cas, le tribunal conclut que la personnalité de la plaignante a teinté sa vision du conflit ${ }^{42}$.

\section{C - LE DROIT DE DIRECTION DE L'EMPLOYEUR}

C'est aussi dans la perspective d'une victime raisonnable que doit être appréciée une conduite reliée à l'exercice du droits de direction. L'abus d'autorité de l'employeur représentait 46\% des plaintes déposées à la CNT entre 2010 et $2013^{43}$. Cette distinction a donc fait l'objet d'une abondante jurisprudence établissant plusieurs principes. D'abord, la jurisprudence énonce que le droit de direction est un pouvoir discrétionnaire qui accorde à l'employeur le droit à l'erreur. En ce sens, l'exercice raisonnable des droits de direction ne constitue pas du harcèlement psychologique au travail. L'exercice déraisonnable des droits de direction par un contrôle arbitraire, abusif et discriminatoire pourra être considéré comme du harcèlement psychologique au travail uniquement lorsque tous les critères constitutifs du harcèlement sont remplis ${ }^{44}$. À titre d'exemple, se faire imposer une évaluation ${ }^{45}$ ou se faire imposer des mesures disciplinaires ${ }^{46}$ ne constituerait pas du harcèlement dans la

37 Roblin c. Commission scolaire Sir-Wilfrid-Laurier/Sir-Wilfrid-Laurier School Board, 2017 OCTAT 3889, par. 557 ; Syndicat de l'enseignement de la Rivière-du-Nord, ibid, §179-181.

38 Laberge, supra note 7, §117; Dubé, supra note 15, §161 (sensibilité de la plaignante).

39 Dubé, ibid, §191.

40 Commission scolaire des Hautes-Rivières et Syndicat du personnel de soutien des Hautes-Rivières, [2012] R.J.D.T. 1005 (T.A.), par. 487, Fixation d'une indemnité, 2014 QCTA 85, Requête en révision judiciaire rejetée, 2015 QCCS 4085.

41 Dian c. Les pêcheries Norrel Québec inc., 2007 QCCRT 0551.

42 Dubé, supra note $15, \S 190$ et 192.

43 C. Belzile et V. Caron, "Profil des salariés ayant déposé une plainte pour harcèlement psychologique », dans Le harcèlement psychologique au travail 2004-2014, De la prévention à la résolution, Yvon Blais, Cowansville, Québec, 2014, p. 1.

44 Dessureault c. La Revue de Terrebonne inc., 2010 QCCRT 0094, §16-18 ; Guimond, supra note 7, $\S 20$; Laberge, supra note 7, par. 153 ; Association des professeur (e)s de Bishop's University, supra note 8, par. 599 et ss ; Bangia c. Nadler Danino, supra note 9, §249-251.

45 Syndicat des chargées et chargés de cours de l'Université de Montréal, supra note 12, §115.

46 CIUSSS de l'Énergie, supra note 30, $\$ 1000$. 
mesure où ces décisions sont justifiées. Toutefois, lorsqu'un employeur s'emporte après un salarié dès qu'il commet une erreur et qu'il a un style de gestion humiliante, il pourrait s'agir de harcèlement psychologique ${ }^{47}$.

En conclusion, les caractéristiques du régime de protection du harcèlement psychologique au travail ont donné lieu à divers enjeux jurisprudentiels liés à l'identification du harcèlement. La jurisprudence démontre que certains comportements, même s'ils peuvent être inappropriés et délétères, ne constitueront pas pour autant du harcèlement psychologique au sens de la LNT.

Ces comportements pourraient toutefois contrevenir au droit à des conditions de travail justes et raisonnables au sens de la Charte des droits et libertés de la personne. D'ailleurs, dans une décision récente ${ }^{48}$, un arbitre a conclu qu'un programme d'optimisation de la performance au travail constituait une violation du droit à des conditions de travail justes et raisonnables en raison de ses répercussions néfastes sur le climat de travail. À l'opposé, des plaintes de harcèlement psychologique ont été rejetées parce que la réorganisation des services ne visait pas uniquement la plaignante mais tout le personnel de l'établissement ${ }^{49}$ ou parce que les objectifs de rendement fixés par l'employeur étaient raisonnables ${ }^{50}$. En fait, contrairement au recours en harcèlement psychologique, une violation du droit à des conditions de travail justes et raisonnables ne requiert pas une conduite vexatoire et ne considère que les conséquences sur le milieu de travail ${ }^{51}$.

47 Franche, supra note 15, §121-125.

48 Alliance du personnel professionnel et technique de la santé et des services sociaux (APTS) c. Centre intégré universitaire de santé et de services sociaux du Nord-de-l'île-de-Montréal, 2016 QCTA 129, Pourvoi en contrôle judiciaire rejeté, 2017 QCCS 1583.

49 Centre intégré universitaire de santé et de services sociaux du Centre-Ouest-de-l'île-de-Montréal, supra note 10, §76.

50 CIUSSS de l'Énergie, supra note 30, par. 830.

51 Pour une analyse de cette décision, voir J.Bourgault, L. Boivin, « Nouvelle gestion publique, action syndicale et défense du droit à des conditions de travail justes et raisonnables », dans J. Grenier et M. Bourque, Le système de santé et des services sociaux québécois. Une analyse de l'évolution des services sociaux et du travail social : idées, institutions, acteurs (titre provisoire), Québec, Presses de I'Université Laval, à paraître. 